Lucrările Seminarului Geografic Dimitrie Cantemir

Vol. 44, October 2017, pp. 159-174

http://dx.doi.org/10.15551/lsgdc.v44i0.11

\title{
Contribution de l'agriculture saharienne a la sécurité alimentaire en Algérie : mythe ou réalité ?
}

\author{
Abdelmadjid Bouder ${ }^{1}$, Tarik Chella ${ }^{1}$ \\ ${ }^{1}$ Université des sciences et de la technologie Houari-Boumédiène, Algérie
}

To cite this article: Bouder, A., Chella, T. (2017). L'aménagement du territoire en Algérie : nouvelle orientation spatiale dans le contexte de libéralisation de l'économie. Lucrările Seminarului Geografic Dimitrie Cantemir, Vol. 44, pp. 159-174. DOI: 10.15551/lsgdc.v44i0.11 To link to this article: http://dx.doi.org/10.15551/lsgdc.v44i0.11 


\title{
CONTRIBUTION DE L'AGRICULTURE SAHARIENNE A LA SECURITÉ ALIMENTAIRE EN ALGÉRIE : MYTHE OU REALITÉ ?
}

\author{
Abdelmadjid Bouder ${ }^{1}$, Tarik Chella ${ }^{2}$
}

Résumé. L'Algérie est dans une situation de fragilité réelle, car elle dépend en ce qui concerne son alimentation des importations couvertes exclusivement par des recettes issues de l'exportation d'hydrocarbures. L'Algérie mise sur le Sahara pour un développement agricole contribuant à assurer une sécurité alimentaire et répondre aux besoins alimentaires de base (céréales et maraichères). Cette communication a pour objectif de montrer le développement acquis dans l'agriculture saharienne (le passage d'une agriculture traditionnelle oasienne, à une agriculture moderne), et la contribution de l'agriculture saharienne à la sécurité alimentaire au niveau national. Cette agriculture qui commence à donner de très bons résultats doit être soutenue avec beaucoup de précautions afin d'éviter de fâcheuses retombées socioéconomiques et écologiques.

Mots clés : L’agriculture saharienne - la sécurité alimentaire - développement agricole Patrimoine oasien.

\section{Introduction}

L'Algérie est située au nord-ouest de l'Afrique en bordure de la Méditerranée. Elle possède une superficie de 2,382 millions $\mathrm{km}^{2}$, ce qui en fait le neuvième pays du monde et le plus grand pays du continent africain. Le relief est constitué de trois grands ensembles : le Tell au Nord, les hauts plateaux et l'Atlas saharien au Centre, et le Sahara au Sud. Mais, paradoxalement, ce pays «continent» peine à garantir la sécurité alimentaire à sa population. La raison en est que la partie apte à l'agriculture, parce qu'elle possède les éléments naturels nécessaires, ne dépasse que rarement le Tell qui ne représente que $4 \%$ du territoire accueillant plus de $70 \%$ de la population totale du pays. Ailleurs, l'agriculture n'est pratiquée que timidement : sur les franges septentrionales des Hauts-Plateaux semi arides on y cultive des céréales à faible rendement ; alors que les parties méridionales sont le domaine exclusif de l'élevage extensif d'ovins. Quant au Sahara, cet immense territoire aride qui couvre environ les trois quarts du territoire national, l'agriculture n'est pratiquée que dans des Oasis au nombre restreint à la faveur de la présence de la ressource en eau de nappes artésiennes jaillissantes.

L’agriculture algérienne qui a longtemps évolué sous ce régime extensif, comptant exclusivement sur le bon vouloir d'une nature au demeurant capricieuse, commençait à atteindre ses limites dès les années 1980 conséquemment à une croissance démographique

\footnotetext{
${ }^{1}$ Professeur, LVRGT, USTHB Alger-Algérie, abouder@yahoo.fr

${ }^{2}$ Maitre de conférences-A-, LVRGT, USTHB Alger-Algérie, chellatarik@yahoo.fr
} 
galopante. En effet, alors que la population a quintuplé depuis l'indépendance du pays, la production agricole, n'a réalisé que de très faibles performances. En conséquence, cette agriculture qui couvrait les besoins de la population et dégageait un surplus à l'exportation quand la population se chiffrait à 12022000 d'habitants en 1966 (ONS, RGPH 1966) est devenue déficitaire dès les années 1990 lorsque la population a dépassé les 20 millions d'habitants (29100867 habitants - ONS, RGPH 1998).

Aujourd'hui, la population du pays est estimée à plus de 40 millions d'âmes et dépend pour plus de $60 \%$ de ses besoins alimentaires de l'importation. Une facture couverte exclusivement par la rente pétrolière aux variations erratiques et peu prévisibles. Le dernier choc pétrolier semble faire prendre conscience aux autorités des enjeux politiques et géostratégiques que fait courir l'insécurité alimentaire pour le pays. La modernisation de l'agriculture implique l'augmentation des surfaces irriguées (de 800000 actuellement à 2000000 ha à l'horizon 2025) et cette dernière est devenue une priorité absolue dans la politique du développement de ces dernières années. Des politiques publiques ambitieuses en matière d'irrigation, d'énergie et de développement d'infrastructures, associées également à l'émergence de dynamiques économiques locales, ont permis un extraordinaire développement de l'agriculture notamment dans les régions sahariennes.

Pour expliciter cette problématique de la sécurité alimentaire en Algérie en général et celle de l'apport de l'agriculture saharienne à cette même sécurité, ce travail de recherche a été initié dans le cadre des constatations faites sur le terrain et à travers des travaux de recherche en cours de lancement. L'importance des résultats initiaux mérite à notre sens d'être d'ores et déjà partagée.

\section{Agriculture et sécurité alimentaire en Algérie}

D'après l'O.N.S, le secteur de l'agriculture emploie près de $25 \%$ de la population active de l'Algérie (ONS, 2008), et contribue à hauteur de $10 \%$ au PIB du pays. Le chiffre d'affaires réalisé par l'industrie agroalimentaire représente $40 \%$ du total du chiffre d'affaires des industries algériennes après celle de l'énergie. Le secteur de l'agriculture est en plein développement car le pays possède tout de même d'importantes potentialités en termes de terres agricoles (Mitidja, Seybouse, Soummam, Touat, Biskra, le Souf, etc.). Cependant, la superficie agricole utile (S.A.U) ne représente que huit millions d'hectares, confinée le long de la façade méditerranéenne et sa largeur ne dépasse que rarement 300 kilomètres à l'intérieur du territoire. Elle ne représente en fait que 3,5\% du territoire et on peut penser qu'il n'existe donc de marge confortable dans l'optique d'extension des terres cultivables. Le reste du territoire est constitué de terres inhospitalières, peu ou pas adaptées à l'agriculture comme on peut le voir avec la figure 1 . 


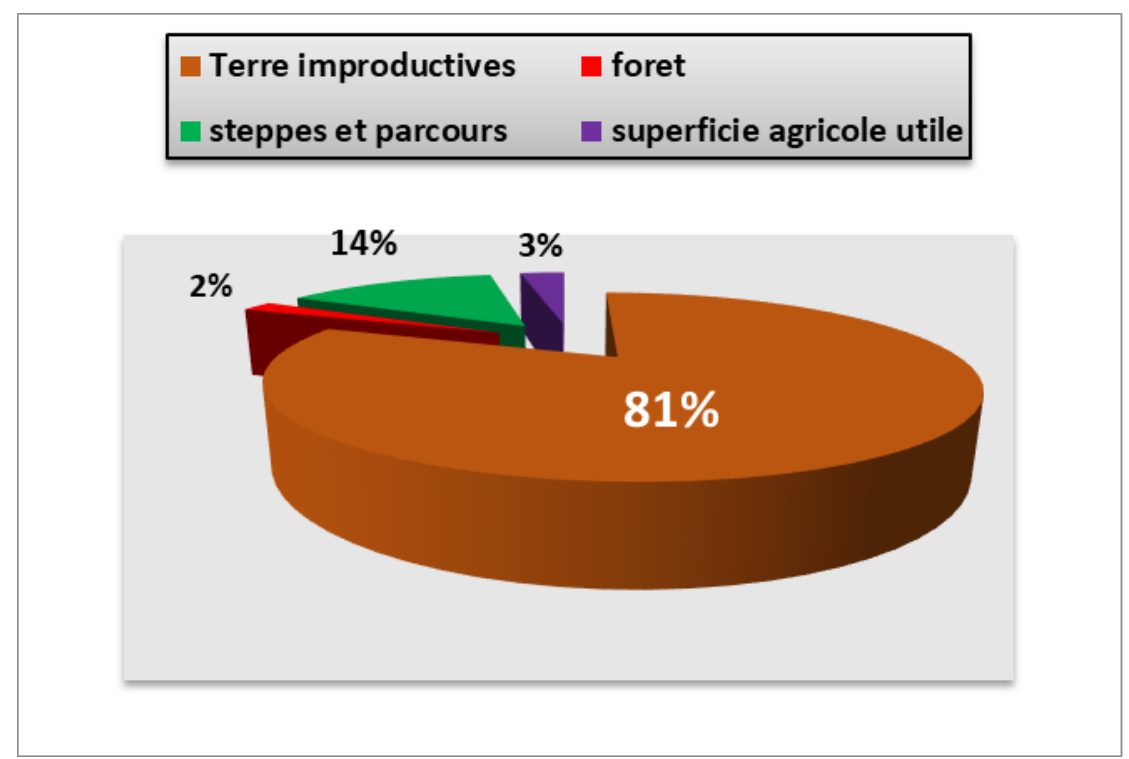

\section{Figure 1 : La Répartition des terres en Algérie Source : MADR, Série B}

La SAU du pays n'a pas connu de variation notable depuis la décolonisation : elle passe de 7,2 millions d'hectares avant 1962, à près de 8,5 millions ha en 2015 (BOUDER A., 2015). La céréaliculture continue d'occuper des superficies identiques à celles d'il y a 53 ans, soit 3,4 millions ha, mais pour des rendements toujours faibles (10Qx/ha en moyenne annuelle) et une production actuelle oscillant entre 45 et 50 millions de quintaux. De prime abord nous constatons que la production de céréales a connu un bond quantitatif appréciable, mais insuffisant par rapport à une croissance démographique très rapide, comme nous le verrons plus loin).

L'agriculture algérienne peut se résumer en quelques indicateurs à savoir :

- $10 \%$ du PIB agricole ;

- 43,4 Millions d'hectares de terres agricoles (17,4\% du territoire) ;

- $\quad$ 8,5 Millions d'ha de Surfaces Agricoles Utiles (S.A.U) ;

- Plus de 1 million d'exploitations agricoles ;

- $\quad 95 \%$ des besoins en machines agricoles proviennent de l'importation ;

- $25 \%$ de la population active employée par ce secteur ;

- Une augmentation de la production agricole depuis le lancement du Plan National de Développement Agricole et Rural (PNDAR) et de la politique de renouveau de l'économie agricole et rurale.

la figure 2 :

La production agricole en Algérie touche beaucoup de filières comme indiqué dans 


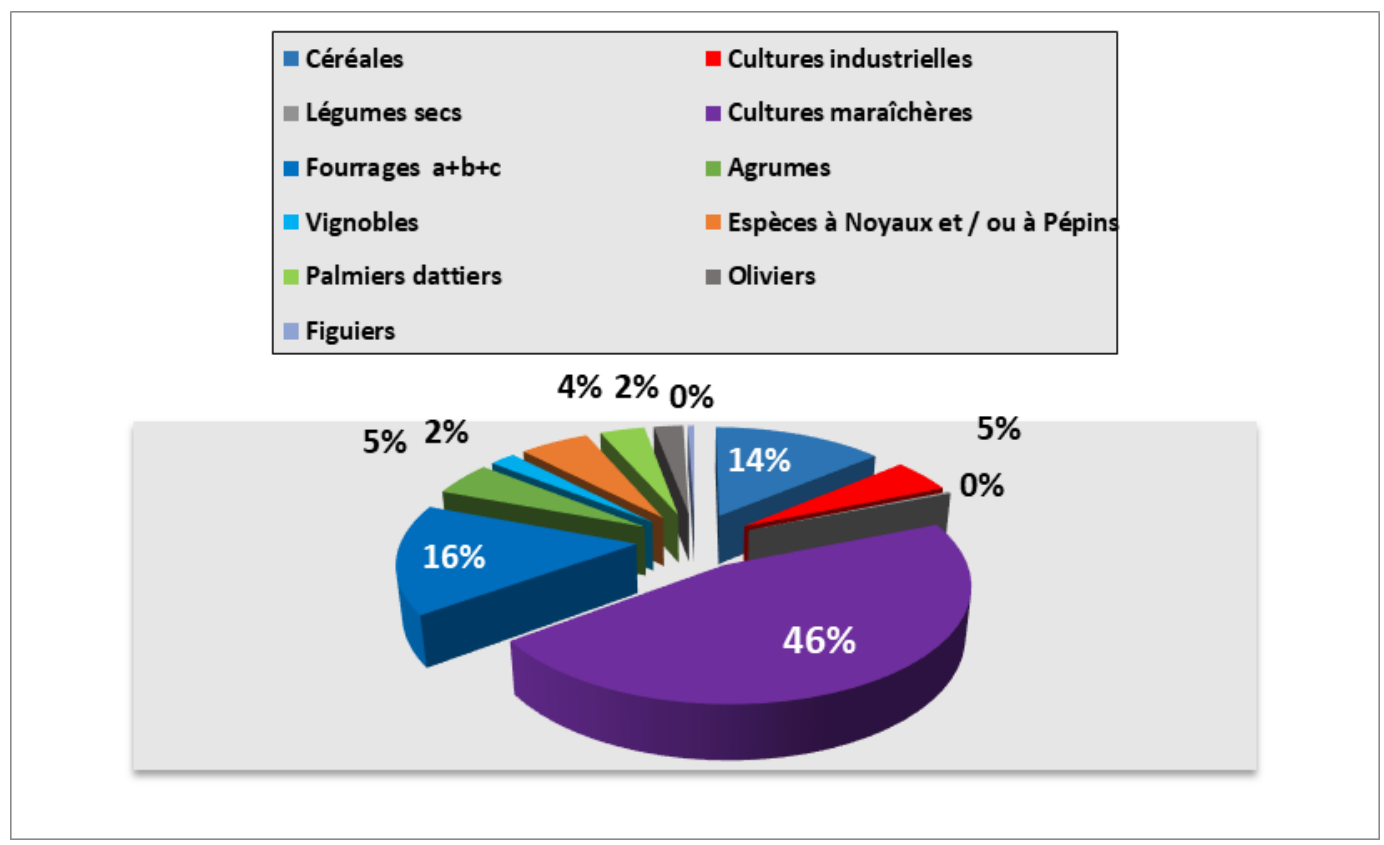

Figure 2 : La production agricole nationale en 2015 selon les filières Source : MADR, Série B

La filière des « cultures maraichères » occupe le premier rang dans la structure de la production agricole nationale réalisée durant l'année 2015, avec une part de $46 \%$ du volume global, en deuxième rang viennent les fourrages avec $16 \%$, les céréales $14 \%$, les espèces à noyaux $6 \%$, et les agrumes $5 \%$. Pour le reste des produits agricoles on remarque une très faible production pour le palmier dattier avec $4 \%$ seulement, en dernier rang les oliviers avec $2 \%$.

L'agriculture algérienne ne remplit pas son rôle qui est celui d'assurer l'alimentation aux autochtones (CNIS/Douanes. 2016 et Ministère du Commerce/statistiques 2016). Durant l'année 2016, les importations des produits alimentaires ont atteint 8,22 milliards de dollars, alors que les prix des produits alimentaires sur les marchés internationaux et la parité Dollar/ Dinar algérien ont baissé. La part des céréales dans cette facture est de 2,81 milliards de dollars en 2016. En effet l'Algérie a importé 12,34 millions de tonnes de céréales, en hausse de $26 \%$ par rapport à 2012. Il est aussi utile de rappeler qu'en 2016 la facture des importations a fait un bond de 4,8 \% par rapport à l'année 2015. Force est de constater que les différentes politiques menées depuis l'indépendance n'ont pas permis d'assurer une indépendance alimentaire.

\subsection{Les produits alimentaires les plus importés par l'Algérie}

L'Algérie est dans une situation de fragilité réelle en matière de production alimentaire qui se situe en deçà des besoins sans cesse croissants, d'où le recours exclusif aux recettes issues des hydrocarbures pour s'approvisionner sur les marchés mondiaux pour des produits qui constituent la base alimentaire des populations. 
Il s'agit des produits céréaliers, des sucres et sucreries, des légumes secs, des produits laitiers, des huiles alimentaires brutes, des cafés et thés, et des viandes. Ces produits représentaient près de $70 \%$ des importations en 2016. Les céréales (semoules et farines), sont les produits qui constituent une part importante dans le modèle de consommation algérien, qui occupe près de $40 \%$ des importations alimentaires en 2016. L'Algérie est l'un des plus grands pays consommateurs de céréales au monde. Les quantités importées de lait sont estimées à 358.943 tonnes durant l'année 2016, soit $12 \%$ des quantités alimentaires importées, et les produits laitiers sont également considérables avec une consommation moyenne de 110 litres de lait par habitant et par an. L'Algérie est le plus important consommateur de lait dans le Maghreb (Tunisie : 83L/hab/an ; Maroc : 64L/hab./an). Les importations de sucre et de ses matières premières (sucres blanc, roux, de betterave brute, de canne à sucre, sirop de lactose...) ont connu une augmentation, se chiffrant à 871,7 millions de dollars (USD) pour l'année 2016, contre 714,76 millions USD en 2015. La même tendance haussière est observée pour les quantités des importations de sucre, qui ont également marqué une augmentation à 2,03 millions de tonnes (Mt) contre 1,93 Mt, selon le Centre national de l'informatique et des statistiques des Douanes (CNIS). Ainsi, le coût des importations de sucre a grimpé de près de $22 \%$ en 2016 tandis que les quantités importées ont augmenté de 5,25\%. Selon le CNIS, cette hausse de la facture du sucre s'explique non seulement par l'augmentation des quantités importées, mais également par le renchérissement des prix sur les marchés internationaux où l'Algérie s'approvisionne. La consommation moyenne de sucre en Algérie est de $24 \mathrm{~kg}$ par habitant et par an. Face à l'absence de culture de canne à sucre et de betterave sucrière, la totalité du sucre brut, de canne ou de betterave, est importée.

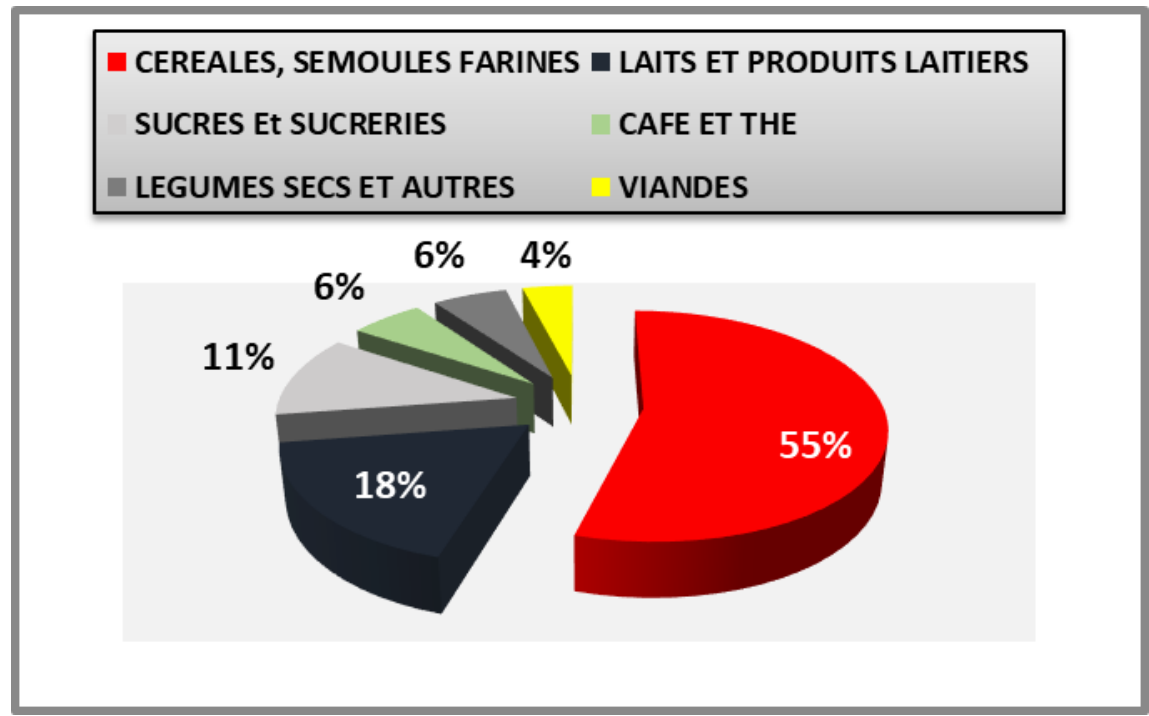

Figure 3: Les produits alimentaires les plus importés en 2015

Source: ONS 


\subsection{Politique récentes de développement agricole en Algérie}

Les insuffisances des politiques antérieures, mises en relief lors des bilans et analyses multiples réalisés en particulier depuis la fin des années 1990, expliquent les déficiences de l'agriculture nationale. Les performances insuffisantes ont conduit, d'abord en 2000, à l'avènement du PNDA (Plan national de développement agricole), puis après une étape d'approfondissement de la réflexion et du renforcement des capacités, est intervenu, en 2009, la politique du Renouveau agricole et rural, sur la base des leçons tirées et de l'identification des bouleversements affectant les diverses composantes du contexte interne et mondial. La réorientation stratégique des politiques agricoles et autres politiques sectorielles liées a permis des progrès tangibles de la sécurité alimentaire nationale. Le niveau de sécurité peut être apprécié par la production et les disponibilités alimentaires.

La croissance en volume de la production agricole, qui a atteint $12,9 \%$ au cours des cinq années de mise en œuvre de la politique de Renouveau Agricole et Rural (2009-2013), a été supérieure à l'objectif initial qui s'élevait à $8,3 \%$. En valeur, la production totale est passée de près de 492 milliards de dinars en 2000 à 2521 milliards de dinars en 2013 (MADR/DSASI/2013). Les mêmes avancées ont été réalisées pour les autres produits (légumes secs, lait), le pays est aujourd'hui parvenu à produire $72 \%$ de ses besoins alimentaires (MADR, 2012). Après avoir pu stopper la tendance à l'aggravation du poste importation de produits alimentaires, l'on s'achemine vers une tendance de redressement. L'analyse de la composition de ces importations (blé dur, blé tendre, orge, légumes secs, lait) révèle, en effet, une nette baisse pour le blé dur et l'orge ainsi qu'une nette augmentation pour le blé tendre, ce qui confirme les changements observés à la fois dans le profil de la production animale - grande consommatrice de céréales - et dans celui du panier alimentaire des ménages. Il faut souligner enfin que la part des importations alimentaires dans les importations globales est restée relativement inchangée, fluctuant dans une fourchette de $16 \%$ à $20 \%$ au cours de la période (Douanes algériennes, séries annuelles).

Une analyse de l'évolution du taux de croissance de la production agricole totale montre qu'après une augmentation très faible entre 1962 et 1980, celle-ci a cru en moyenne annuelle d'environ $3 \%$ entre 1981 et 1990, de 3,2\% entre 1991 et 2000 et de 7,3\% entre 2001 et 2010 (MADR/DSASI). Cet accroissement qui s'est poursuivi en 2013 met en lumière le dynamisme soutenu que connait le secteur agricole, particulièrement depuis 2009. Les volumes de production de la plupart des produits ont doublé ou triplé (pomme de terre, tomate industrielle, légumes secs, fourrages,...).

Toutefois, cette évolution est également marquée par des variations qui demeurent sensibles d'une année à l'autre $(-7,4 \%$ en $2008,+31,5 \%$ en 2009$)$ et des disparités entre les régions. La diminution des effets des aléas climatiques comme le rattrapage des performances des zones en retrait figurent parmi les objectifs clés affichés.

\subsection{Contribution de l'agriculture saharienne à la sécurité alimentaire}

En Algérie, les régions sahariennes disposent d'un territoire immense. Ces régions disposent aussi de ressources hydriques importantes constituées en majorité de réservoirs d'eau souterraine non renouvelable. Depuis la découverte de ce grand potentiel hydrique et la baisse des coûts de sa mobilisation, le Sahara, où existait une agriculture oasienne en 
difficulté, est considéré comme l'espoir de l'agriculture algérienne. L'accès massif à l'eau souterraine a, dans certaines régions, profondément modifié le paysage des oasis, passant dans certaines régions d'une agriculture oasienne traditionnelle à étages, à des cultures irriguées par pivot ou, dans d'autres régions, à une agriculture combinant des palmiers en monoculture et des serres tunnels à perte de vue.

La mise en valeur au Sahara Algérien se présente même, comme la solution pour la réduction de la dépendance alimentaire du pays. En effet, ses potentialités immenses ont permis d'espérer de pouvoir sortir l'agriculture saharienne de son carcan oasien traditionnel pour s'orienter vers une agriculture moderne sur de nouvelles terres gagnées sur le désert.

Actuellement plusieurs régions du pays sont approvisionnées en fruits et légumes à partir du Sahara, notamment en hiver. L'agriculture saharienne connaîtra dans les années à venir un bond qualitatif au regard des efforts consentis par l'Etat et le génie local. On y recense diverses cultures réussies, des dattes à la pomme de terre, en passant par la céréaliculture, les légumes, les olives, les cacahuètes et les pistaches.

En Algérie, le mythe d'un eldorado agricole saharien ne date pas d'aujourd'hui, il a déjà conduit, dès les années 1980 (Tomate d'Adrar), à des expériences de mise en valeur à grande échelle. Une deuxième vague de concessions agricoles dans le Sud est en cours depuis quelques années sur une échelle encore plus importante. Elle crée déjà la polémique. Elle mérite au moins d'être mise en débat: Cette agriculture est-elle un mythe ou une réalité ?

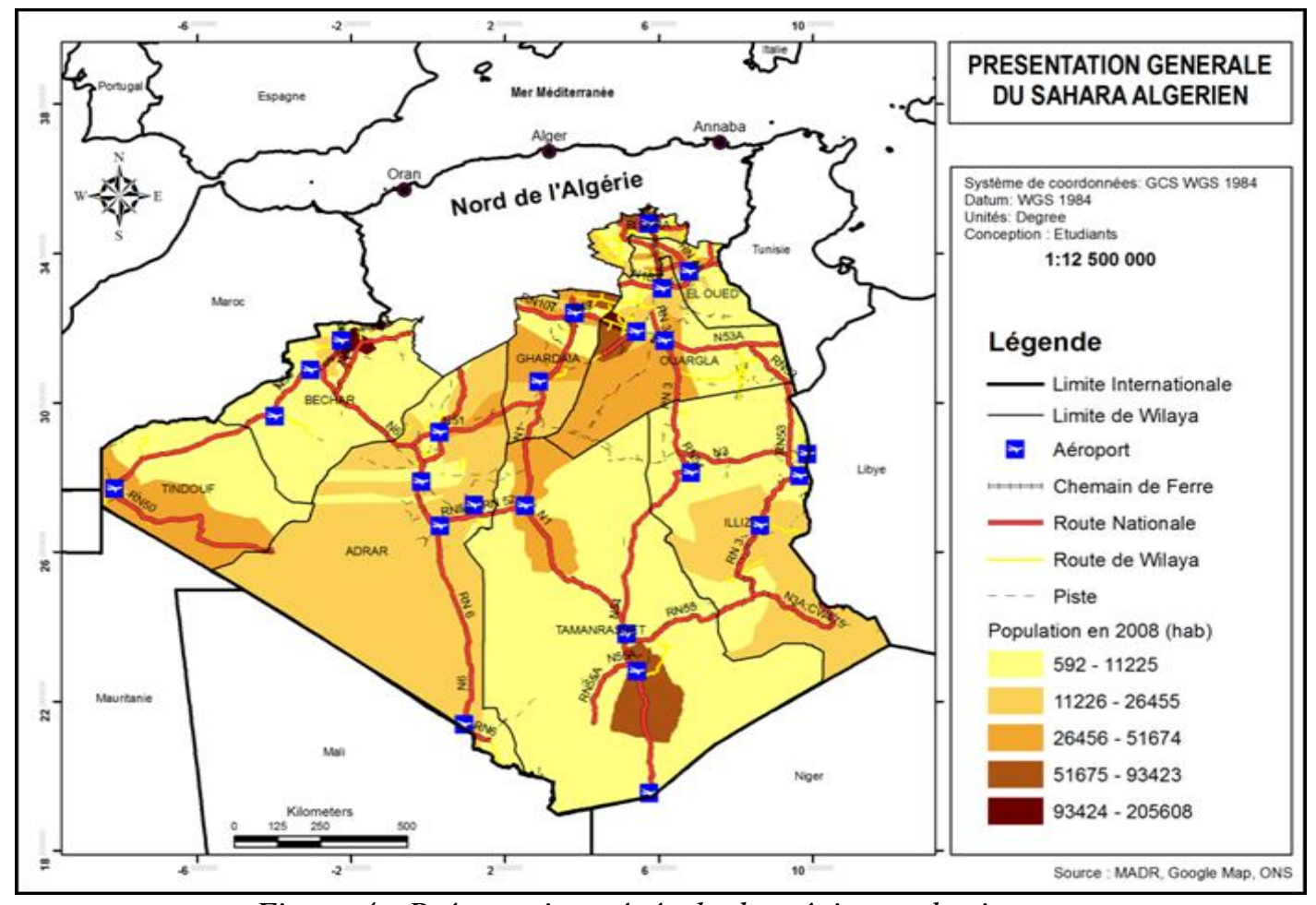

Figure 4 : Présentation générale des régions sahariennes 


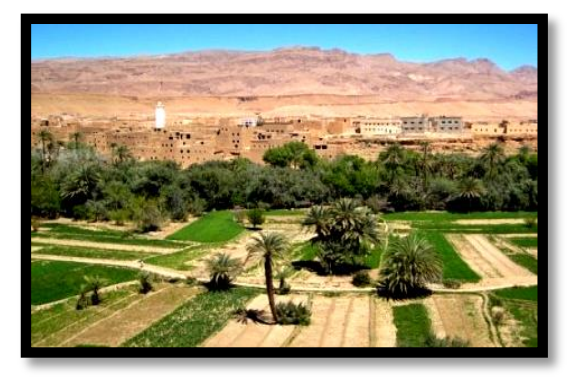

Figure 5 : culture traditionnelle à étages

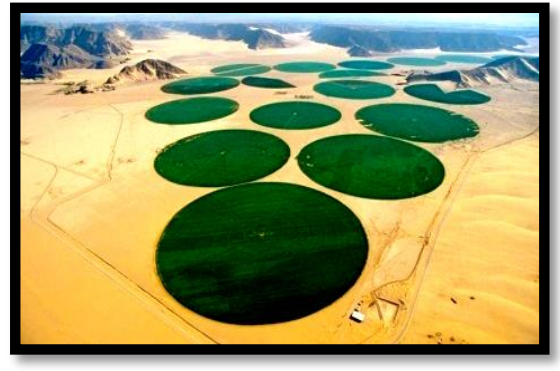

Figure 6: culture moderne à pivots

L'autosuffisance alimentaire a constitué l'un des principaux objectifs de la politique agricole prônée par l'Algérie dès les premières années de l'indépendance, et 50 ans après, elle a commencé avec succès à exploiter les énormes gisements de l'agriculture saharienne. Pour mettre fin à la dépendance alimentaire vis-à-vis de l'étranger, la solution consiste, d'après DUBOST, à encourager l'agriculture dans le sud «Le Sahara est l'avenir de l'Algérie », dit-il avec beaucoup de conviction. De son avis, toutes les conditions d'une agriculture performante sont réunies dans cette région du pays : « Il y a la terre, l'eau et la lumière, soit les trois éléments essentiels à toute culture. » De son avis, l'agriculture saharienne a «tout naturellement » connu un boom extraordinaire ces dernières années. « À Biskra par exemple, il y a d'immenses surfaces qui sont cultivées » (DUBOST D, 1987).

L'agriculture saharienne n'est plus au stade de projet, c'est une réalité tangible. Aujourd'hui, plusieurs régions du pays sont approvisionnées en fruits et légumes depuis Biskra, El Oued, Ouargla et Adrar. Pour autant, l'autosuffisance alimentaire ne sera pas facile à atteindre : la terre, l'eau et la main-d'œuvre, entre autres, posent problème au niveau des régions à fort potentiel agricole. L'agriculture saharienne ne manque pas d'atouts. Le Sud regorge de surfaces cultivables qui ne demandent qu'à être exploitées. L'agriculture saharienne peut contribuer de manière significative à la réduction de la facture alimentaire, mais elle nécessite la mise en place d'une véritable stratégie avec la mobilisation de moyens financiers et surtout de cadres techniques de haut niveau.

\section{Pourquoi le Sahara?}

La priorité accordée au développement de l'agriculture au Sahara s'explique d'abord par la rareté des terres agricoles arables sur la bande littorale du pays à cause du relief montagneux et le rétrécissement de la SAU sous l'effet dévastateur de la croissance urbaine par étalement grignotant chaque année des centaines, voire des milliers d'hectares : Alger et Blida ainsi que la multitude des agglomérations qui les entourent, auraient consommé à nos jours « un peu plus de 120000 ha de la plaine de Mitidja (A. BOUDER, 2015).

A cela s'ajoute la rareté relative et l'inadéquation de la répartition géographique des potentialités en eau et en sol : le réservoir en eau de surface de l'Algérie se trouve à l'Est, un territoire montagneux à plus de $70 \%$, d'où la rareté des terrains arables, alors que les territoires de l'Ouest qui dispose d'un potentiel sol important est situé dans un domaine climatique subaride. La solution préconisée et déjà mise en œuvre consiste à mobiliser l'eau à l'Est et la transférer vers les plaines de l'Ouest sur des distances variant entre 800 et 1000 $\mathrm{km}$. 
Cependant, malgré le bien-fondé de cette solution, ses effets bénéfiques escomptés à savoir la mobilisation de 12 milliards de $\mathrm{m} 3$ au lieu des 6 milliards actuels, ne commenceront à se manifester qu'à long terme (2030), et ce lorsque le nombre de barrages passera de 75 actuellement à 139 à la date indiquée (El Watan du 07/08/2017). Cette solution est donc trop couteuse en investissements et en temps. Devant l'urgence, les regards se sont dirigés vers le Sahara pour son potentiel énorme en eau souterraine.

\subsection{Potentialités et contraintes du Sahara}

Si les regards se sont tournés vers le Sahara dans ces moments de crise c'est parce que celui-ci, nonobstant les contraintes communes à tous les déserts du Monde, possède les potentialités aussi bien naturelles qu'humaines nécessaires au développement agricole. Le plus important est l'existence d'un potentiel hydrique substantiel. Il s'agit du système aquifère du Sahara septentrional couvrant une superficie de $1019000 \mathrm{Km}^{2}$ au total dont $70 \%$ en Algérie, 22\% en Libye et 8\% en Tunisie. En l'an 2000, les prélèvements totaux sur le SASS étaient évalués à 2,5 milliards de m3/an, dont 1,45 pour l'Algérie, 0,55 en Tunisie et 0,5 en Libye, l'exploitation pour l'agriculture reste prédominante dans les trois pays (CRSTRA, 2002).

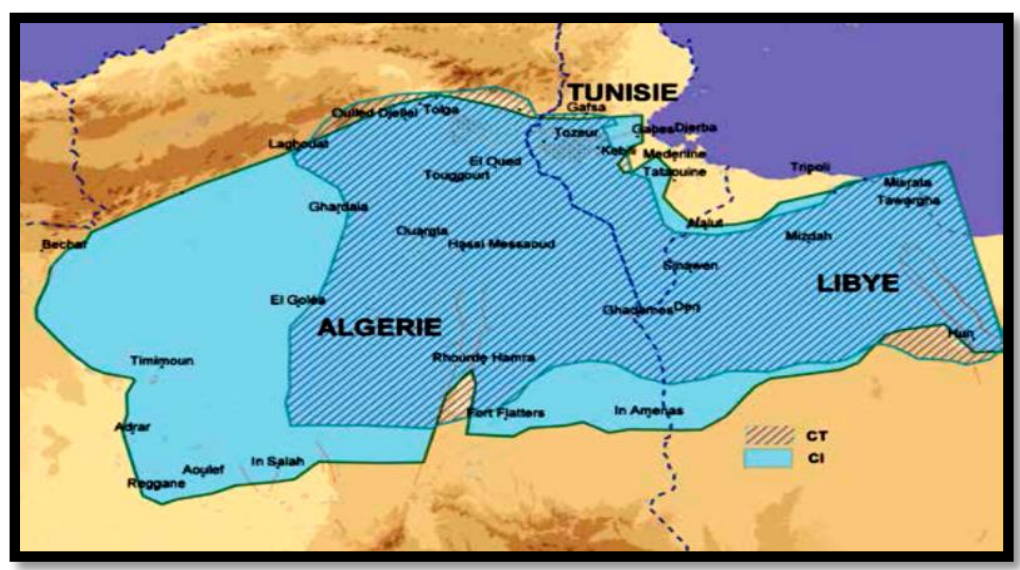

Figure 7 : Le Système aquifère du Sahara Septentrionale (CT et CI) Source : CRSTRA, 2002

D'après les études du PDGDRS, les ressources en eau souterraines au Sahara algérien couvrent une surface de $600000 \mathrm{Km}^{2}$ et renferment 50000 milliards de m3 d'eau en réserve. Elles sont essentiellement constituées par (voir tableau $\mathrm{n}^{\circ} 1$ ci-dessous) :

- Les eaux renouvelables localisées dans les inféro-flux du versant sud des Aurès (Région Nord de Biskra), du Hoggar Tassili à l'Est et de la région de Bechar Tindouf à l'Ouest.

- Les eaux non renouvelables représentées par les deux grands réservoirs des deux bassins sédimentaires : le Complexe Terminal et le Continental intercalaire. 
Tableau 1: Bilan général de l'eau au Sahara algérien (en mètre cube par seconde)

(CRSTRA, 2002)

\begin{tabular}{|l|l|l|l|}
\hline Aquifère & Utilisée en 1990 & \multicolumn{2}{|c|}{ Utilisée en 2000 } \\
\hline & & Hypothèse basse & Hypothèse haute \\
\hline Continental intercalaire & 18,7 & 23,4 & 33,2 \\
\hline Complexe terminal & 15 & 18 & 25 \\
\hline Inferoflux & 16 & 21 & 26 \\
\hline Total & 49,7 & 62,4 & 84,2 \\
\hline
\end{tabular}

A ces quantités d'eau souterraine s'ajoutent d'autres quantités moins importantes constituées par les eaux de surface touchant plus particulièrement les marges septentrionales du Sahara qui se caractérisent par :

- un volume total écoulé entre 540 à $970 \mathrm{Mm}^{3} / \mathrm{an}\left(15.9 \mathrm{~m}^{3} / \mathrm{s}\right)$;

- Et une série de collinaires de piémont d'une capacité totale de $15 \mathrm{Mm}^{3}$;

- 6 barrages offrant $140 \mathrm{Mm}^{3}$ de régularisation annuelle.

Quant aux potentialisés humaines, il y a lieu de savoir que le Sahara algérien est loin d'être un no mans land, car la découverte puis la mise en exploitation des immenses réserves de pétrole et- du gaz dans les années 1950 ont eu pour conséquences directes la rapide transformation des anciens Ksours en de véritables villes dotées de tous les équipements et infrastructures nécessaires.

La plupart des wilayas sahariennes enregistrent un taux de croissance démographique annuel de plus de $2 \%$, dépassant ainsi la performance nationale qui était de 1,6\% en 2008 (ONS, 2008). Ainsi, la population totale de ces wilayas est passée de 900000 hab. en 1967 à plus de 3 millions d'âmes en 2008 comme indiqué par le graphique ci-après. Des villes comme Hassi Missaoud (60000 hab.) et Hassi R'mel (22000 hab.) doivent leur existence à l'avènement de l'activité pétrolière.

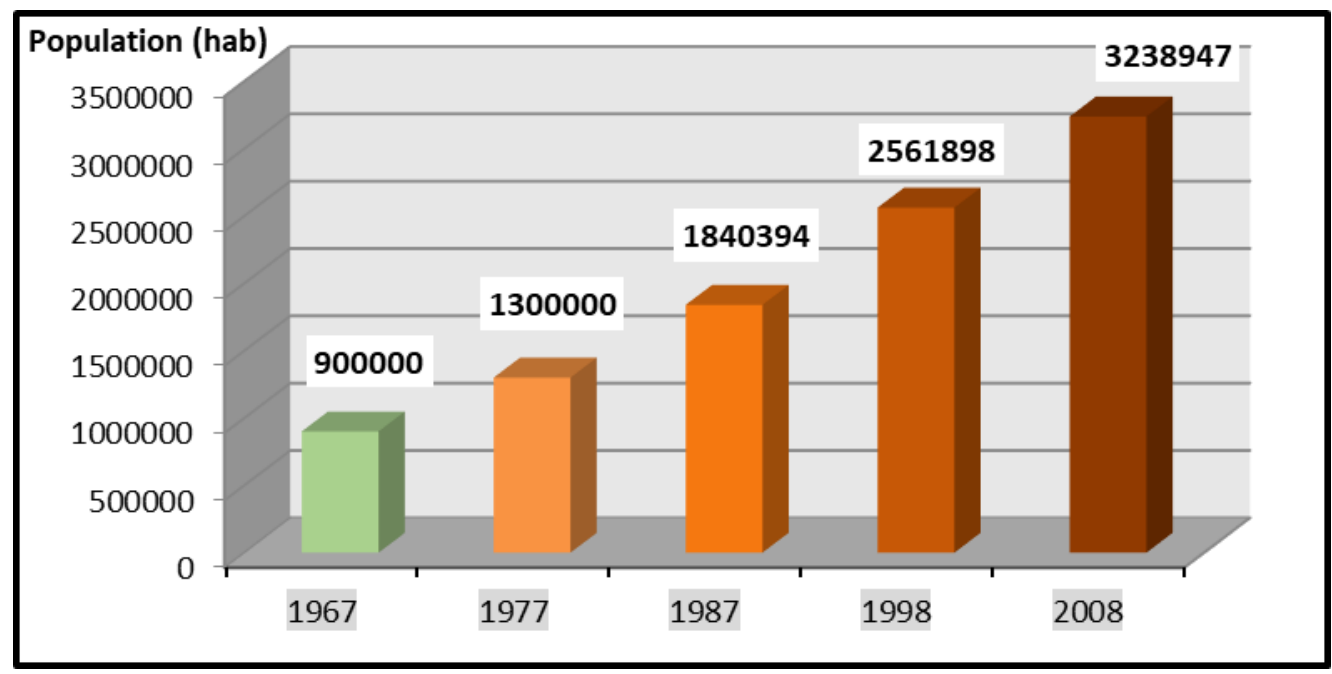

Figure 8: Croissance démographique dans les wilayas du Sahara algérien entre 1967 et 2008 Source : ONS, RGPH 2008 


\subsection{Acteurs et moyens mis en œuvre}

L'agriculture saharienne a connu une profonde évolution à travers différents programmes agricoles depuis l'indépendance, initiés par les pouvoirs publics. Le plus important dans les régions sahariennes est le plan national de développement agricole et rural (PNDAR). Selon (BOUKELLA M. 2008), Ce plan est un vaste programme qui a été lancé dès 1999 sous un titre de (PNDA), devenu en 2002 (PNDAR), dont les objectifs ont été définis pour inclure :

- La consolidation de la contribution à la sécurité alimentaire ;

- La valorisation de toutes les ressources disponibles ;

- La protection de l'environnement.

Etant le promoteur principal du projet, l'Etat, met en œuvre une série de mesures : allégements fiscaux, soutien au développement de la productivité et de la qualité ainsi que la promotion du partenariat avec des opérateurs nationaux et étrangers. Pour ce faire, Les pouvoirs publics ont mobilisé près de 400 milliards de dinars algériens entre 2000 et 2007, à travers un dispositif combinant trois sources de financement :

- Le Fond National de Développement et de Régulation (FNDRA) à hauteur de $60 \%$ du coût total des projets ;

- Le Fond de Mise en Valeur de Nouvelles Terres par la Concession (FMVC) ;

- Le Crédit Agricole Mutuel par le biais de la Banque Algérienne de Développement Rural (BADR).

En conséquence, tous ces efforts n'ont pas tardé à donner leurs fruits sur le terrain : la superficie agricole utile (SAU) a atteint 432670 ha soit $5 \%$ de la SAU nationale, ce qui correspond à une croissance de $63 \%$ depuis 2000 , cette augmentation se vérifiant notamment dans les wilayas de Biskra, El Oued, Adrar, Ouargla, Bechar et Ghardaïa (MADR, Série B). Ces effets se font remarquer également dans le domaine de la production.

\subsection{La production agricole saharienne}

Le Sahara a connu des évolutions rapides dans les années récentes au gré des différents projets de mise en valeur publique et de l'initiative privée qui ont modifié considérablement les données de l'agriculture dans certaines wilayas. Cette évolution est bien approchée par l'indicateur relatif aux attributions APFA (l'Accession à la Propriété Foncière Agricole). Les valeurs indiquées ci-dessous proviennent des annuaires statistiques par wilayas dans la région saharienne. Pour l'agriculture saharienne on ne peut pas se baser seulement sur la filière dattière, il faut élargir la fonction de production à d'autres produits agricoles, voire toute la production de biens et de services et d'autres secteurs d'activité. De toute évidence, il n'était pas possible de développer le Sahara par la seule production de dattiers et l'exploitation de la "Deglet Nour». 


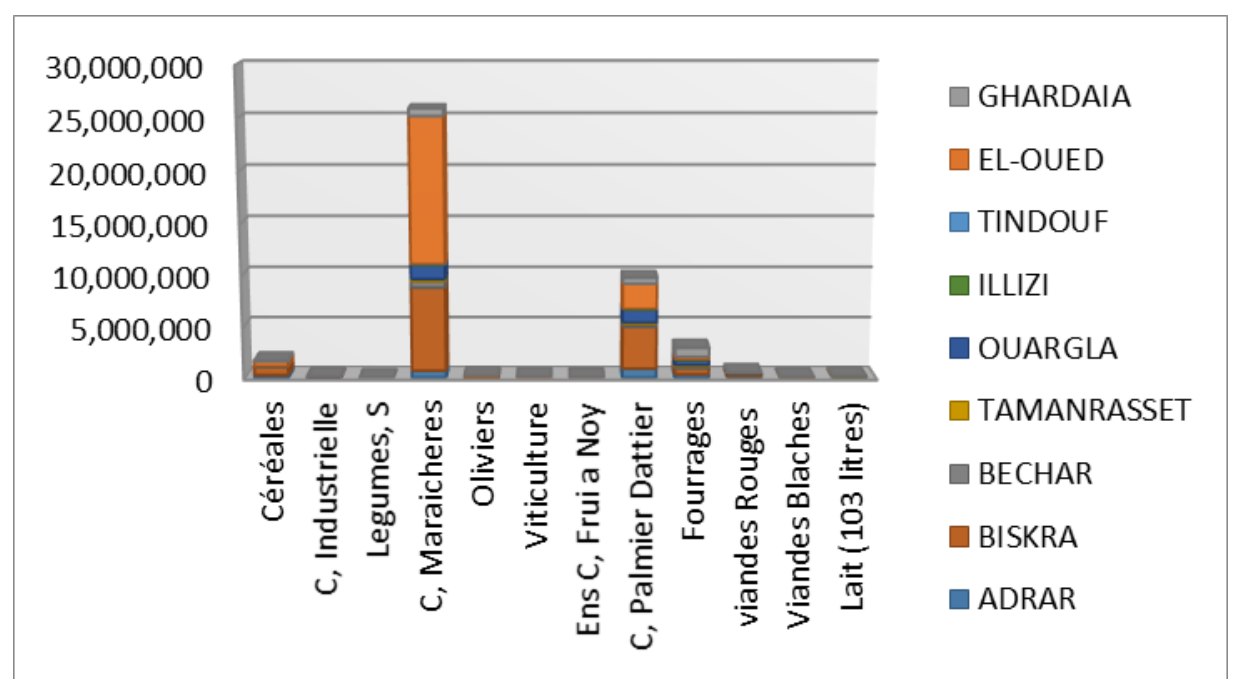

Figure 9 : Production agricole dans la région saharienne par wilaya en (2015) Source : MADR, Série B

D'après le (figure 9) on remarque une forte production agricole dans les wilayas de Biskra, El Oued, Ouargla, Adrar et Ghardaïa, avec une forte production dans le maraichage, céréales et fourrages, mais l'en remarque une légère diminution de la production des dattes comparativement aux années précédentes.

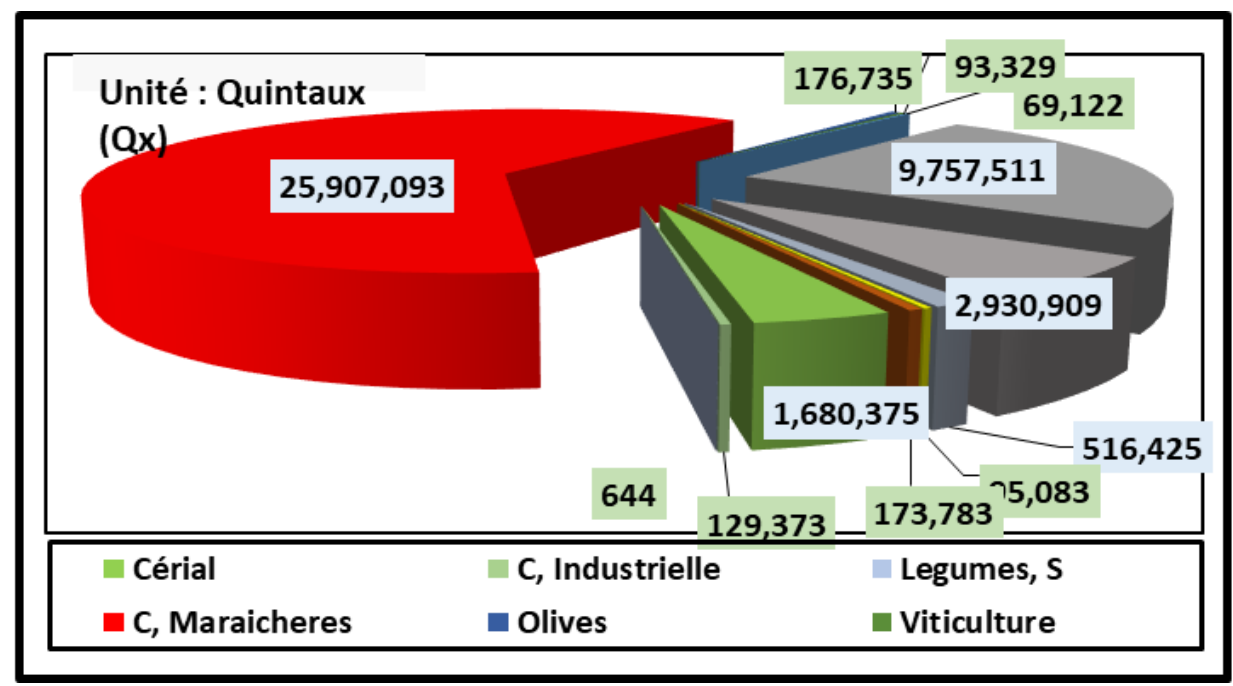

Figure 10: Production agricole dans la région saharienne par filière en 2015 Source : MADR, Série B

Le suivi des surfaces et des productions totales met en évidence une nette progression, la production maraichère occupe le premier rang dans la région saharienne en 2015, avec 25907093 Quintaux (Qx). Les wilayas d'El-Oued, Adrar et Ghardaïa ont connu la pression la plus importante, mais la wilaya de Biskra reste de loin la plus importante en 
surface et en production. Il est clair que le fort développement des cultures sous serres explique que la production continue à croitre avec une meilleure productivité.

Les dattes occupent le deuxième rang de la production agricole saharienne avec 9 757511 Qx. La culture phoenicicole est localisée beaucoup plus dans les wilayas de Biskra, El Oued et Ouargla. On remarque une production importante de fourrages qui occupent le $3^{\text {ème }}$ rang avec 2930909 Qx. La production céréalière au Sahara, introduite au cours de la décennie 1985-1995, atteint désormais une production de 1680375 Qx/an, venant principalement des wilayas de Biskra et El Oued.

Les viandes rouges sont concentrées dans les wilayas de Biskra, Ghardaïa en plus d'Adrar avec une production de 516 425Qx.

Les cultures industrielles, le tabac, le henné et le safran sont des cultures sahariennes significatives à l'échelon national avec 129373 Qx. Pour le reste des productions agricoles sahariennes : Olives, viticulture, ensemble des fruits, laite et légumes secs, la production est très faible, comme le montre la figure 10.

\section{La contribution du Sahara à la Production Agricole Nationale}

L'Algérie mise sur les gisements de l'agriculture saharienne pour assurer son autosuffisance alimentaire. L'agriculture saharienne constitue une sérieuse option pour l'Algérie dans sa quête d'une autosuffisante alimentaire. Son développement est susceptible, en effet, de mettre fin à la dépendance alimentaire en matière des produits agricoles.

La figure 11 ci-dessous exprime la part de la région saharienne dans la production agricole nationale.

La phoeniciculture algérienne est bien entendu entièrement représentée par les domaines sahariens. La culture du palmier dattier offre une diversité et un potentiel variétal inestimable.

Il a été recensé plus de 750 variétés (parmi lesquelles la variété la plus connue qui est la Deglet Nour qui présente la meilleure valeur marchande). Elle occupe la première place en termes de participation agricole nationale avec un pourcentage remarquable de $98.52 \%$, ceci dans des régions bien définies principalement l'Oued Righ, le Ziban et le Souf.

Pour la culture marâichère avec une surface tous légumes confondus de 67259 ha et une production de plus de 2590709 tonnes en 2015, le Sahara a produit $20.77 \%$ de la production nationale.

Les viandes rouges participent avec un taux de $10,8 \%$. Les fourrages sont aussi récoltés dans la zone et représentent $6.08 \%$ de la production nationale.

La production céréalière nationale s'établissant à 37609485 Qx, le Sahara a produit $4.4 \%$ de cette production en 2015 . La production laitière dans ces régions n'est pas non plus absente de la production nationale $(4.89 \%)$, tout comme la fruiticulture $(4.9 \%)$. Pour le reste des cultures, on remarque une faible participation à la production nationale, légumes secs $(0.07 \%)$, culture industrielle $(1 \%)$ et Oliviers $(2.7 \%)$ contribuent peu à la production de leur filière. 


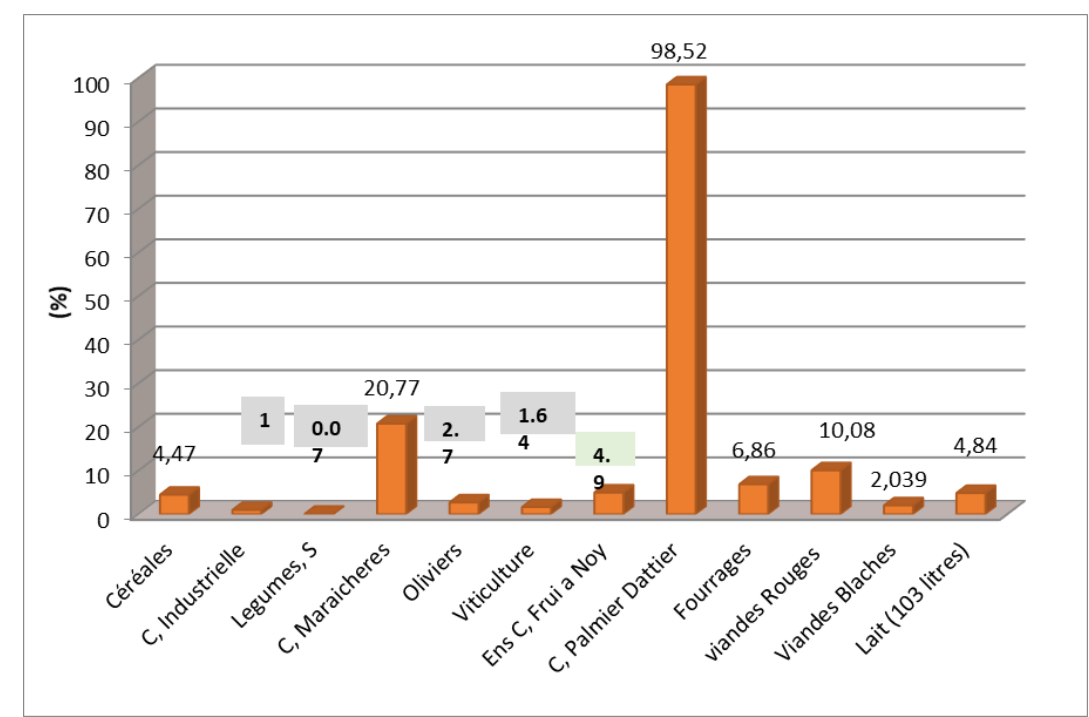

Figure 11 : Taux de participation agricole de la région saharienne à la production nationale de chaque filière, en 2015 (\%)

Source : MADR, Série B

Cette faiblesse de production pour ces dernières cultures dans la région saharienne est due à plusieurs contraintes : stockage, transport et absence d'unités de transformation (pour les cultures industrielles) et de climat, sol et ressources en eaux (pour les légumes secs et les oliviers).

\section{Conclusion : L'agriculture saharienne est-elle durable ?}

L'introduction de l'agriculture moderne au Sahara est considérée aujourd'hui comme un impératif économique majeur, car c'est un palliatif des problèmes dont souffre l'agriculture algérienne dans les territoires du Nord. Le développement de cette agriculture saharienne ne peut être envisagé qu'en relation avec le développement global de l'agriculture algérienne.

Il ne peut en aucun cas être vu comme un substitut. En effet, une telle agriculture pratiquée dans un milieu fragile avec des facteurs de production exclusivement artificiels, comptant sur une eau fossile non renouvelable dont l'exploitation excessive induirait des risques écologiques désastreux, ne peut garantir le même degré de durabilité que l'agriculture du Nord possède intrinsèquement.

Il faut donc réduire les ardeurs de ceux qui pensent que l'avenir de l'agriculture algérienne est au Sud d'autant plus que les spécialistes parlent d'un seuil d'exploitation déterminé : «on peut escompter aujourd'hui que l'agriculture saharienne dispose d'environ $50 \mathrm{~m} 3 / \mathrm{s}$ d'eau d'irrigation $(. .$.$) cette eau prise globalement permettrait de cultiver de 60$ à 80000ha »(DUBOST D., 2002). Aller au-delà c'est mettre en péril le patrimoine oasien ancestral par la baisse du niveau piézométrique des nappes phréatiques ainsi que l'élévation du degré de salinité des eaux. 


\section{Référencés bibliographiques}

1. Bouder A., Chella T., 2015. L'étalement urbain sur les terres agricoles comme obstacle majeur à la sécurité alimentaire en Algérie, cas de l'extension d'Alger et de Blida sur la plaine de la Mitidja, International Journal for Environment and Global Climate Change, Vol. 3, Issue 2

2. Bouder A., 2015. Impact de l'urbanisation sur l'étalement urbain à Alger : esquisse d'un bilan, in villes et métropoles algériennes, revue du GREMAMO n²2, L’Harmattan, Paris.

3. Boukella M., 2008. Politique agricole, dépendance et sécurité alimentaire, in l'Algérie de demain, relever les défis pour gagner l'avenir, Friedrich Ebert Stiftung.

4 .Cnis, Douanes, 2017. Alger, statistiques du commerce extérieur de l'Algérie, année de 2016.

5. Dubost D., 1987. Nouvelles perspectives agricoles du Sahara algérien, Revue de l'Occident musulman et de la Méditerranée,No. 41-42, 339-356 ;

6. Dubost D., 2002. Ecologie, aménagement et développement agricole des oasis algériennes, CRSTRA, Biskra.

7. El Watan, 2017. Alger.

8. Hamiche M., Hammouda M., Meziani A., 2017. Contribution de l'agriculture saharienne à la sécurité alimentaire en Algérie, cas de la filière de pomme de terre dans la wilaya d'El Oued, Master en aménagement régional et compétitivité territoriale, USTHB.

9. https://www.commerce.gov.dz/statistiques.

10. MADR, Direction des statistiques Agricoles et des Systèmes d'Information, série (B), Alger, 2012, 2013, 2014, 2015;

11. ONS, RGPH, Alger, 1966, 1998, 2008 ;

12. ONS, statistiques agricoles, Alger, 2008 ;

13. Centre de Recherche Scientifique et Technique sur les Régions Arides (CRSTRA), Biskra, 2002. 
